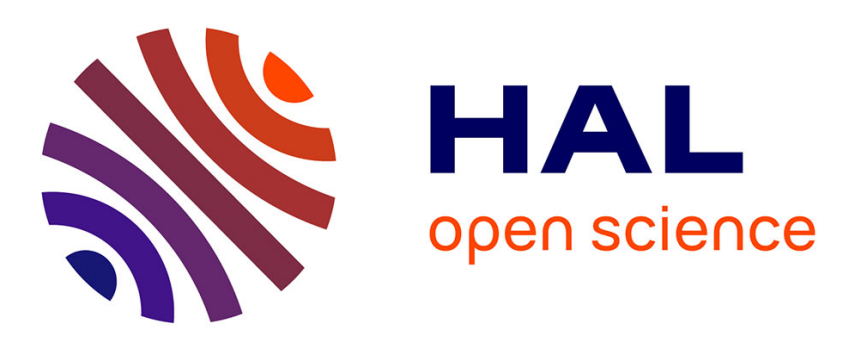

\title{
An Improved Method for the Geometrical Calibration of Parallelogram-based Parallel Robots
}

\author{
Ludovic Savoure, Patrick Maurine, David Corbel, Sébastien Krut
}

\section{To cite this version:}

Ludovic Savoure, Patrick Maurine, David Corbel, Sébastien Krut. An Improved Method for the Geometrical Calibration of Parallelogram-based Parallel Robots. ICRA: International Conference on Robotics and Automation, May 2006, Orlando, United States. pp.769-776, 10.1109/ROBOT.2006.1641803 . lirmm-00118225

\section{HAL Id: lirmm-00118225 \\ https://hal-lirmm.ccsd.cnrs.fr/lirmm-00118225}

Submitted on 4 Dec 2006

HAL is a multi-disciplinary open access archive for the deposit and dissemination of scientific research documents, whether they are published or not. The documents may come from teaching and research institutions in France or abroad, or from public or private research centers.
L'archive ouverte pluridisciplinaire HAL, est destinée au dépôt et à la diffusion de documents scientifiques de niveau recherche, publiés ou non, émanant des établissements d'enseignement et de recherche français ou étrangers, des laboratoires publics ou privés. 


\title{
An Improved Method for the Geometrical Calibration of Parallelogram-based Parallel Robots
}

\author{
Ludovic Savoure $^{(1)}$, Patrick Maurine ${ }^{(1)}$, David Corbel ${ }^{(2)}$, Sébastien Krut ${ }^{(2)}$ \\ (1) INSA de Rennes, Laboratoire GCGM \\ 20 Avenue des Buttes de Coësmes, CS 14315 \\ 35043 RENNES CEDEX, FRANCE \\ patrick.maurine@insa-rennes.fr
}

\begin{abstract}
This paper presents an improved method for the geometrical calibration of parallel robots for which the structure is based upon some parallelogram mechanisms. Its originality is to identify the complete geometry of the mechanism's parallelograms, and to compensate the positioning error of the TCP (Tool Centre Point), due to the infinitesimal rotation of the traveling plate, induced by the parallelogram geometrical errors. The main difficulties are: (i) to derive the calibration model relative to all geometrical parameters, and (ii) to reuse the identified errors in a control model in order to compensate the positioning errors. In fact, the position relationship taking into account the full geometry of the parallelograms is difficult, not to say impossible, to derive in a close form; therefore a linear approximation of the model is proposed. The formulas necessary to run the method on a Delta robot are given. Then a simple mechanism is used to illustrate the benefits of this method compared to classical ones.
\end{abstract}

Keywords: Geometrical Calibration, Compensation, PKM, Deltalike Parallel Robots, Spatial parallelograms

\section{INTRODUCTION}

Nowadays, Parallel Kinematic Machines (PKM) are more and more involved in new applications within domains such as highspeed machining, pick and place or medical. However, improving the accuracy of these machines is still a challenge and intensive research works have already been done on this subject [1]. Those works show that the technique of the calibration is usually involved in order to increase the PKM dexterity in terms of accuracy.

The calibration is an economic process by which the positioning accuracy of a machine can be improved without modifying its physical structure. It consists in using redundant sensory information combined with dedicated algorithms in order to perform the identification as well as the compensation of the most relevant errors that affect, in terms of accuracy, the model running on the machine controller. That sensory information can be obtained by using external sensors, by adding extra sensors on the mechanism structure or by restraining the TCP motion through some locking devices. For the two latter solutions, the process is then viewed as self-calibration and an interesting summary of all these calibration techniques is available in [2]. According to the nature of the identified and compensated errors, the calibration can be categorized as geometrical, elastostatic, thermal and dynamic [3][4][5]. The integration within the calibration model of those different error sources as well as the parameter identification complexity depends on the machine structure and process.

This is in this PKM calibration context that our research takes place. Since the middle of the 90s, researchers have been intensively working on the development and advancement of new calibration methods suitable for all existing PKM structures. Most of these methods concern the calibration and compensation of the geometrical effects that reduce the static positioning accuracy. It is also to be underlined that more recent works have focussed on the non-geometrical effects which degrade also the accuracy as for example the static and dynamical elastic deformations [6][7][8].

The research work presented in this paper proposes a new calibration method that allows to improve the positioning accuracy of a family of PKM for which the structure is made with spatial parallelograms (parallelograms made of rods connected with spherical joints). As examples of robots belonging to that family one can note, the 3 degrees-of-freedom (dof) ABB FlexPicker, a commercialized version of the Delta robot [9], the H4 robot developed at the LIRMM [10], the 6-dof 321-HEXA [11].

As it will be shown in the following, for this PKM family, only the geometrical errors that affects the positioning accuracy will be identified and compensated. The non-geometric factors such as flexibility of the structure beams and gear train, the gear backlashes, the encoder resolutions errors, the thermal effects as well as the dynamic effects have not been considered. In other words, the proposed method is a geometrical calibration approach for parallelogram-based PKM that can be involved to improve their static positioning accuracy.

This method has been developed according to the following assessment that can be drawn up: the major part of the calibration methods which have been already proposed in the literature for parallelogram-based PKM are limited to identifying the geometrical parameters involved in the control models, i.e. the position relationships linking the TCP position to the actuated joint values [12][13][14][15]. However for those parallel robots, the control models are simplified. They rely on the hypotheses that the parallelograms are perfect (the rods of a pair are exactly of the same length). Doing so leads to major simplifications (the travelling plate remains always parallel to the ground) and allows writing most of the control models in a close form.

Yet the parallelograms are not perfect (as a consequence of manufacturing and assembling errors) resulting in infinitesimal rotations of the travelling plate leading to positioning errors of the TCP. This phenomenon is accentuated the grater the distance between the TCP and the median plane of the travelling plate is, as this distance amplifies the effects of the infinitesimal rotations. Compensating the positioning errors thanks to the simplified control models does not ensure to compensate this phenomenon (because of lack of modelling).

In order to find a possible solution to this problem, the approach that is proposed in this paper is to use models involving the 
complete geometry of the robot (including the exact geometry of the parallelograms) for the identification and the compensation process. The main difficulty when considering the exact geometry of the robot is to derive the position relationships. Hence no simplification can be done (the travelling plate does not remain necessarily parallel to the ground), and writing the control models in a close form becomes difficult, not to say impossible. In this paper we propose to use an approximation of the position relationships: the first order linear estimation (relatively to geometrical errors). Thanks to it, we are able to compensate the positioning errors, using the identified full geometry of the mechanism.

Section 2 describes the proposed calibration method. At first, the way to identify the full geometry of a Delta robot is given and the hypotheses relative to the calibration process are recalled. Then, the way to derive the first order linear approximation of the position relationship is given, in order to make the positioning error compensation taking into account the identified complete geometry. The next section illustrates the benefits of the proposed method thanks to a simple mechanism. This mechanism is simple enough to derive all the required models in a close form, and complex enough to witness the same properties of parallelogrambased parallel robots. Comparing the positioning errors after classical and improved calibration shows the benefits of the proposed method. Some extensions to this work are given in last section, before concluding.

\section{DESCRIPTION OF THE PROPOSED CALIBRATION METHOD}

The geometrical calibration process of robots consists of four steps well-known as: (i) modelling, (ii) measurement, (iii) identification and (iv) compensation [16]. The compensation process relies on the use of the position relationships updated with the geometrical parameters estimated during the identification phase. As a consequence there is a strong correlation between those two phases.

For Delta-like parallel robots the position relationships are simplified, involving some virtual geometrical lengths (the lengths of virtual rods located at the middle each parallelogram). Hence, for classical calibration, the identification process does not seek the exact geometry of the robot but estimates these virtual geometrical lengths.

To our knowledge, very few complete models, taking into account the geometry of the 6 rods for a Delta robot, have been used for identification and compensation [13]. This can be explained by the significant increase of the mathematical complexity of the identification step [13][17] but also to the real difficulties resulting in using straightforwardly the identified geometrical lengths, in the TCP position relationships involved during the compensation phase. The proposed calibration method relies on a complete description of the mechanism (involving the exact geometry of the parallelograms) for identification and compensation. However because it is difficult, not to say impossible, to derive the position relationships in a close form for this family of robot, an approximation of the model, simple enough to be computed in a close form, is proposed.

The following table (Table 1) summarizes the names of all the models involved during calibration. We will call "complete" [C index] (by opposition to "simplified" [ $S$ index]) the models involved in the proposed method. Notations are explained bellow. Explanations will be given in the next sections.

TABLE 1

\begin{tabular}{|c|c|c|c|c|}
\hline \multicolumn{5}{|c|}{ MODELS GLOSSARY } \\
\hline & & & Simplified models & Complete models \\
\hline \multirow{4}{*}{\begin{tabular}{|l|} 
Control \\
models
\end{tabular}} & \multirow{2}{*}{\begin{tabular}{|l|l|} 
Position \\
relations
\end{tabular}} & Forward & $\boldsymbol{x}=\boldsymbol{F P R _ { S }}\left(\boldsymbol{q}, \boldsymbol{k}_{S}\right)$ & $\boldsymbol{x}=\boldsymbol{F P R _ { C }}\left(\boldsymbol{q}, \boldsymbol{k}_{C}\right)$ \\
\hline & & Inverse & $q=I P R_{S}\left(x, k_{S}\right)$ & $\boldsymbol{q}=\widetilde{\boldsymbol{I P R _ { C }}}\left(\boldsymbol{x}, \boldsymbol{k}_{C}\right)$ \\
\hline & \multirow{2}{*}{\begin{tabular}{|l|} 
Velocity \\
relations
\end{tabular}} & Forward & $\dot{\boldsymbol{x}}=\boldsymbol{J}_{S}\left(\boldsymbol{q}, \boldsymbol{k}_{S}\right) \dot{\boldsymbol{q}}$ & Not required \\
\hline & & Inverse & $\dot{\boldsymbol{q}}=\boldsymbol{J}_{S}^{m}\left(\boldsymbol{q}, \boldsymbol{k}_{S}\right) \dot{\boldsymbol{x}}$ & Not required \\
\hline $\begin{array}{l}\text { Identificatio } \\
n \text { models }\end{array}$ & \multicolumn{2}{|c|}{ Sensibility matrix } & $\delta \boldsymbol{x}=\boldsymbol{S}_{S}\left(\boldsymbol{q}, \boldsymbol{k}_{S}\right) \delta \boldsymbol{k}_{S}$ & $\delta \boldsymbol{X}=\boldsymbol{S}_{C}\left(\boldsymbol{q}, \boldsymbol{k}_{C}\right) \delta \boldsymbol{k}_{C}$ \\
\hline
\end{tabular}

$\boldsymbol{k}$ designates a vector made with the geometrical parameters to be identified. $\boldsymbol{x}$ is the vector of operational coordinates and $\boldsymbol{q}$ stands for joint coordinates. $\dot{\boldsymbol{x}}$ and $\dot{\boldsymbol{q}}$ designate the operational and joint velocities. (This glossary concerns a Delta robot more particularly; hence, $\boldsymbol{x}$ concerns displacements only, and $\dot{\boldsymbol{x}}$ is its time derivative.) $\delta$ is used to mention small values. Thus $\delta \boldsymbol{k}$ designate the errors on the geometrical parameters and $\delta \boldsymbol{x}$ the difference between the theoretical position of the TCP and its real position once measured. To do the calibration using the complete model, the position and the orientation of the travelling plate need to be measured. Hence we introduce:

$$
\delta \boldsymbol{X}^{\mathrm{T}}=\left[\begin{array}{ll}
\delta \boldsymbol{x}^{\mathrm{T}} & \delta \boldsymbol{\alpha}^{\mathrm{T}}
\end{array}\right]_{1 \times 6},
$$

where $\delta \boldsymbol{\alpha}$ represents the orientation error.

Matrices $\boldsymbol{J}_{S}, \boldsymbol{J}_{S}^{m}, \boldsymbol{S}_{S}$ and $\boldsymbol{S}_{C}$ depend on the geometrical parameters of the robot $\left(\boldsymbol{k}_{S}\right.$ or $\left.\boldsymbol{k}_{C}\right)$ and on the posture of the mechanism. The joint coordinates $\boldsymbol{q}$ were chosen to express this dependence; however in a practical manner, the matrices are also defined using $\boldsymbol{x}$, as $\boldsymbol{x}$ is not necessarily written explicitly using $\boldsymbol{q}$. As the simplification done when computing the position relationships, is at the origin of the difference between the simplified and the complete calibration method, this point will be detailed in the following. Then the way to make the complete calibration for a Delta robot will be given.

A. The way to derive the position relationships and the usual hypotheses done for simplication purpose

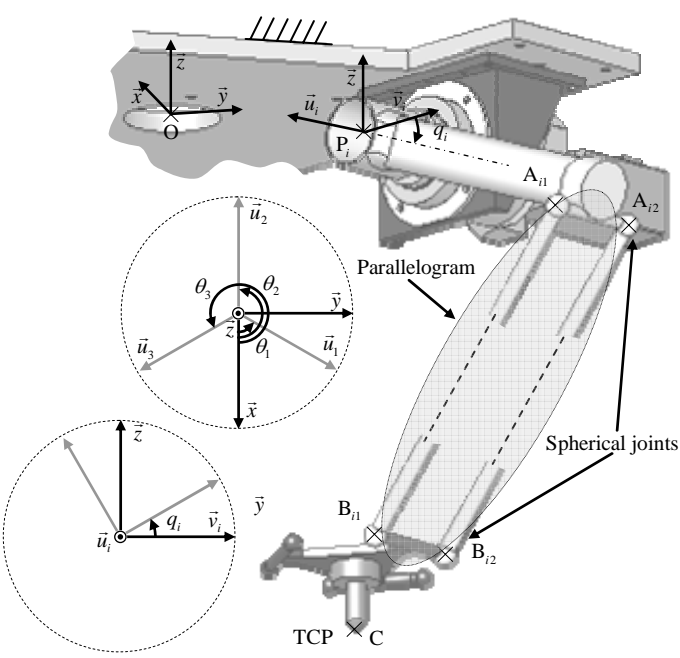

Fig. 1 - Scheme of a pair of rods of the Delta robot 
Deriving the position relationships for a Delta robot is done when characterizing the coupling between the traveling plate (located in space using $\boldsymbol{x}$ ) and the actuated arms (defined using $\boldsymbol{q}$ ), exerted by the rods. Hence each rod, connected with spherical joints, adds a unique constraint between these two sets: The distance between the parts connected to the extremities of the rods equals the lengths of the rods. Mathematically this corresponds to (see Fig. 1):

$$
{\overrightarrow{\mathrm{A}_{i j} \mathrm{~B}_{i j}}}^{2}=l_{i j}{ }^{2}, i \in\{1,2,3\}, j \in\{1,2\}
$$

where points $\mathrm{A}_{i j}$ are defined thanks to $\boldsymbol{q}$, and $\mathrm{B}_{i j}$ thanks to $\boldsymbol{x}$.

B. Identification of the geometrical parameters using a complete geometrical model

1) The complete model used during identification

As usually done for calibration, the model used to identify the errors on geometrical parameters is approximated: it is obtained when differentiating an expression mapping all the variables of the system ( $\boldsymbol{x}, \boldsymbol{q}$ and $\boldsymbol{k}$ ) relatively to all its terms, and making the linear approximation. This approximation consists in assuming that the errors to be computed are small enough to be assimilated to the derivative terms:

$$
\delta \boldsymbol{k} \simeq d \boldsymbol{k}, \delta \boldsymbol{q} \simeq d \boldsymbol{q}, \delta \boldsymbol{x} \simeq d \boldsymbol{x} .
$$

For parallelogram-based robots, instead of using the position relationship (forward or inverse) as the relation mapping all the variables, one prefers to use directly the raw system of equations (2). Hence, even if the position relationships cannot be derived in a close form, this model can. The differential of (2) is:

$$
\overrightarrow{\mathrm{A}_{i j} \mathrm{~B}_{i j}} \cdot d \overrightarrow{\mathrm{A}_{i j} \mathrm{~B}_{i j}}=l_{i j} d l_{i j} \text {. }
$$

Starting from this equation, we will see below how to express in a compact manner the identification model. Once written in the canonic basis $\mathcal{B}=(\vec{x}, \vec{y}, \vec{z})$, and making the linear approximation (3), relation (4) becomes:

$$
\mathbf{A}_{i j} \mathbf{B}_{i j}{ }^{\mathrm{T}}\left(\delta \mathbf{B}_{i j}-\delta \mathbf{A}_{i j}\right)-l_{i j} \delta l_{i j}=0 \text {. }
$$

T denotes the matrix transposition, $\delta \mathbf{B}_{i j}$ is a column vector representing the position error of point $\mathrm{B}_{i j}$ (same for $\delta \mathbf{A}_{i j}$ ), and $\delta l_{i j}$ is the error to be identified on length $l_{i j}$.

Making the small displacements assumption, $\delta \mathbf{B}_{i j}$ can be written:

$$
\delta \mathbf{B}_{i j}=\delta \boldsymbol{x}+\widehat{\mathbf{B}_{i j} \mathbf{C}} \delta \boldsymbol{\alpha}+\delta \boldsymbol{b}_{i j}
$$

where $\widehat{\mathbf{B}_{i j} \mathbf{C}}$ represents the cross-product matrix:

$$
\widehat{\mathbf{B}_{i j} \mathbf{C}} \delta \boldsymbol{\alpha}=\mathbf{B}_{i j} \mathbf{C} \times \delta \boldsymbol{\alpha}
$$

and $\delta \boldsymbol{b}_{i j}$ represents position errors of point $\mathrm{B}_{i j}$ relatively to the traveling plate. In the same way:

$$
\delta \mathbf{A}_{i j}=\delta \boldsymbol{p}_{i}+\boldsymbol{R}_{i} \delta \boldsymbol{a}_{i j}+\widehat{\mathbf{A}_{i j} \mathbf{P}_{i}} \delta \boldsymbol{\beta}_{i} \text {, where: }
$$

$\square \delta \boldsymbol{p}_{i}$ is the position error of point $\mathrm{P}_{i}$ relatively to the ground, $\square \delta \boldsymbol{a}_{i j}$ the position error of point $\mathrm{A}_{i j}$ relatively to the arm,

$\square \boldsymbol{R}_{i}$ represents the rotation of amplitude $q_{i}$ around the axis orientated according to vector $\boldsymbol{u}_{i}$ (see Fig. 1),

$\square \delta \boldsymbol{\beta}_{i}$ is a vector corresponding to the angular errors witnessing the misalignment and the offset errors of the revolute actuators.
Matrix $\boldsymbol{R}_{i}$ can be spit as follows:

$$
\boldsymbol{R}_{i}=\boldsymbol{P}_{i} \boldsymbol{Q}_{i}
$$

where $\boldsymbol{P}_{i}$ is the matrix mapping the main basis $\mathcal{B}$ into the local basis $\mathcal{B}_{i}=\left(\vec{u}_{i}, \vec{v}_{i}, \vec{z}\right)$ (see Fig. 1 ):

$$
\boldsymbol{P}_{i}=\left[\begin{array}{ccc}
\cos \theta_{i} & -\sin \theta_{i} & 0 \\
\sin \theta_{i} & \cos \theta_{i} & 0 \\
0 & 0 & 1
\end{array}\right]
$$

$\theta_{1}=60^{\circ}, \theta_{2} \in 180^{\circ}$ and $\theta_{3}=300^{\circ}$ correspond to the orientation of each actuator relatively to the frame. $\boldsymbol{Q}_{i}$ is the rotation matrix of amplitude $q_{i}$ written in the local basis $\mathcal{B}_{i}$ (see Fig. 1):

$$
\boldsymbol{Q}_{i}=\left[\begin{array}{ccc}
1 & 0 & 0 \\
0 & \cos q_{i} & -\sin q_{i} \\
0 & \sin q_{i} & \cos q_{i}
\end{array}\right]
$$

Hence, relation (5) written in a synthetic manner becomes:

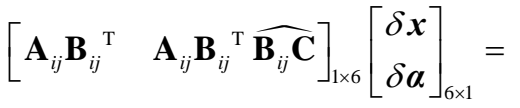

$$
\begin{aligned}
& {\left[\begin{array}{lll:l}
\mathbf{A}_{i j} \mathbf{B}_{i j}{ }^{\mathrm{T}} & \mathbf{A}_{i j} \mathbf{B}_{i j}{ }^{\mathrm{T}} \boldsymbol{R}_{i} & l_{i j} & \mathbf{A}_{i j} \mathbf{B}_{i j}{ }^{\mathrm{T}} \widehat{\mathbf{A}_{i j} \mathbf{P}_{i}}
\end{array}\right]_{1 \times 10}\left[\begin{array}{c}
\delta \boldsymbol{b} \boldsymbol{p}_{i j} \\
\delta \boldsymbol{a}_{i j} \\
\delta l_{i j} \\
\hdashline \delta \boldsymbol{\beta}_{i}
\end{array}\right]_{10 \times 1}}
\end{aligned}
$$$$
\text { where: } \quad \delta \boldsymbol{b} \boldsymbol{p}_{i j}=\delta \boldsymbol{b}_{i j}-\delta \boldsymbol{p}_{i}
$$

Let us note:

$$
\begin{gathered}
\boldsymbol{J}_{k C_{i j}}^{a}=\left[\begin{array}{lll}
\mathbf{A}_{i j} \mathbf{B}_{i j}{ }^{\mathrm{T}} & \mathbf{A}_{i j} \mathbf{B}_{i j}{ }^{\mathrm{T}} \boldsymbol{R}_{i} & l_{i j}
\end{array}\right]_{1 \times 7}, \\
\boldsymbol{J}_{k C_{i j} b}^{b}=\left[\begin{array}{lll}
\mathbf{A}_{i j} \mathbf{B}_{i j}{ }^{\mathrm{T}} & \widehat{\mathbf{A}}_{i j} \mathbf{P}_{i}
\end{array}\right]_{1 \times 3} \text { and: } \\
\delta \boldsymbol{k}_{C_{i j}{ }^{\mathrm{T}}}^{\mathrm{T}}=\left[\begin{array}{lll}
\delta \boldsymbol{b} \boldsymbol{p}_{i j}{ }^{\mathrm{T}} & \delta \boldsymbol{a}_{i j}{ }^{\mathrm{T}} & \delta l_{i j}{ }^{\mathrm{T}}
\end{array}\right]_{1 \times 7} .
\end{gathered}
$$

Writing relation (12) for all the six rods $(i, j) \in\{(1,1),(1,2),(2,1),(2,2),(3,1),(3,2)\}$, and merging the results together leads to:

$$
\boldsymbol{J}_{X} \delta \boldsymbol{X}=\boldsymbol{J}_{k C} \delta \boldsymbol{k}_{C}
$$

with: $\quad \boldsymbol{J}_{X}=\left[\begin{array}{cc}\mathbf{A}_{11} \mathbf{B}_{11}{ }^{\mathrm{T}} & \mathbf{A}_{11} \mathbf{B}_{11}{ }^{\mathrm{T}} \widehat{\mathbf{B}_{11} \mathbf{C}} \\ \vdots & \\ \mathbf{A}_{32} \mathbf{B}_{32}{ }^{\mathrm{T}} & \mathbf{A}_{32} \mathbf{B}_{32}{ }^{\mathrm{T}} \widehat{\mathbf{B}_{32} \mathbf{C}}\end{array}\right]_{6 \times 6}$,

$$
\begin{aligned}
& \boldsymbol{J}_{k C}=\left[\begin{array}{ccc:ccc} 
& & & \boldsymbol{J}_{k C_{11}}^{b} & \mathbf{0} & \mathbf{0} \\
& & & \boldsymbol{J}_{k C_{12}}^{b} & \mathbf{0} & \mathbf{0} \\
\boldsymbol{J}_{k C_{11}}^{a} & & \mathbf{0} & \mathbf{0} & \boldsymbol{J}_{k C_{21}}^{b} & \mathbf{0} \\
& \ddots & & 0 & \boldsymbol{J}_{k C_{22}}^{b} & \mathbf{0} \\
\mathbf{0} & & \boldsymbol{J}_{k C_{32}}^{a} & \mathbf{0} & \mathbf{0} & \boldsymbol{J}_{k C_{31}}^{b} \\
& & & 0 & \mathbf{0} & \boldsymbol{J}_{k C_{32}}^{b}
\end{array}\right]_{6 \times 51}
\end{aligned}
$$

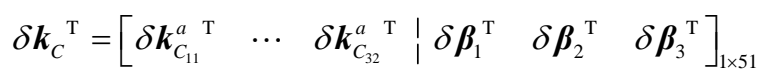

Hence the sensibility matrix mapping the position error of the TCP $\delta \boldsymbol{X}$ to the errors on geometrical parameters $\delta \boldsymbol{k}_{C}$ is obtained: 
$\begin{array}{ll} & \delta \boldsymbol{X}=\boldsymbol{S}_{C}\left(\boldsymbol{q}, \boldsymbol{k}_{C}\right) \delta \boldsymbol{k}_{C} \\ \text { with: } \quad & \boldsymbol{S}_{C}\left(\boldsymbol{q}, \boldsymbol{k}_{C}\right)=\boldsymbol{J}_{X}^{-1} \boldsymbol{J}_{k C} .\end{array}$

$$
\boldsymbol{S}_{C}\left(\boldsymbol{q}, \boldsymbol{k}_{C}\right)=\boldsymbol{J}_{X}{ }^{-1} \boldsymbol{J}_{k C} \cdot
$$

The identification method using this matrix is recalled below.

2) Recall of the identification method

As on the one hand the non-geometrical effects are not considered in this study and on the other hand the parallelogram-based PKM structures are assumed perfectly rigid, the previously defined sensibility matrix $\boldsymbol{S}_{C}\left(\boldsymbol{q}, \boldsymbol{k}_{C}\right)$ will be involved in the identification step. $\boldsymbol{S}_{C}\left(\boldsymbol{q}, \boldsymbol{k}_{C}\right)$ maps according to (21), the differential errors on the geometrical parameters $\delta \boldsymbol{k}_{C}$ and the resulting differential static positioning error of the TCP, $\delta \boldsymbol{X}$. To identify $\delta \boldsymbol{k}_{C}$, equation (21) will be applied by using a sufficient number $m$ of robot configurations $\boldsymbol{q}_{k}$ and the corresponding TCP positions and orientations will be measured to calculate

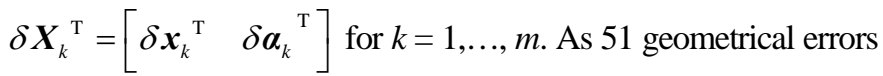
have to be identified, at least $m=9$ robot configurations have to be used for the measurements since relation (21) gives 6 mathematical relations for each configuration. The resulting number of relations will be represented by the following equation system by using a global sensitivity matrix $\boldsymbol{S}_{G}$ defined as follows:

$$
\delta \boldsymbol{X}_{G}=\boldsymbol{S}_{G} \delta \boldsymbol{k}_{C}
$$

where: $\delta \boldsymbol{X}_{G}=\left[\begin{array}{c}\delta \boldsymbol{X}_{1} \\ \vdots \\ \delta \boldsymbol{X}_{m}\end{array}\right]$ and $\boldsymbol{S}_{G}=\left[\begin{array}{c}\boldsymbol{S}_{C}\left(\boldsymbol{q}_{1}, \boldsymbol{k}_{C}\right) \\ \vdots \\ \boldsymbol{S}_{C}\left(\boldsymbol{q}_{m}, \boldsymbol{k}_{C}\right)\end{array}\right]$

It is to note that if the involved measuring system does not allow measuring the orientation $\delta \boldsymbol{\alpha}_{k}$ for each configuration $k$, only the relations corresponding to the position error can be used as explained in [18]. The identification is then achieved by using equation (23) that is solved in order to obtain the least-squares solution of the geometrical parameter errors.

\section{Identifiability of the geometric errors}

The determination of the base of identifiable parameters $\delta \boldsymbol{k}_{C}^{b}$ has to be performed before solving the linear system of equations (23). This set of parameters is classically obtained numerically by using the QR decomposition of the global sensitivity matrix $\boldsymbol{S}_{G}$ as explained in [18]. This allows to obtain the reduced sensitivity matrix $\boldsymbol{S}_{G}^{b}$ which contains the $b$ independent and non-null columns of $\boldsymbol{S}_{G}$ corresponding to the parameters errors $\delta \boldsymbol{k}_{C}^{b}$. This leads to writing the reduced linear system of equation (25) from which the identification is derived.

$$
\delta \boldsymbol{X}_{G}=\boldsymbol{S}_{G}^{b} \delta \boldsymbol{k}_{C}^{b}
$$

The least squares solution $\widehat{\delta \boldsymbol{k}_{C}^{b}}$ of (25) can be obtained by using $\boldsymbol{S}_{G}^{b+}$, the pseudo inverse matrix of the global sensitivity matrix

$\boldsymbol{S}_{G}^{b}$ as follows: $\widehat{\delta \boldsymbol{k}_{C}^{b}}=\boldsymbol{S}_{G}^{b+} \delta \boldsymbol{X}_{G}$

As $\boldsymbol{S}_{G}^{b}$ is a full rank matrix the computation of $\boldsymbol{S}_{G}^{b+}$ is given by $\left(\boldsymbol{S}_{G}^{b \mathrm{~T}} \boldsymbol{S}_{G}^{b}\right)^{-1} \boldsymbol{S}_{G}^{b \mathrm{~T}}$. Note that the standard deviation of the estimated geometrical errors can be calculated by using the matrix $\boldsymbol{S}_{G}^{b}$ [19].

\section{Approximation of the Complete Inverse Position Relationship used for the compensation}

To compensate the position error of the TCP due to errors on geometrical parameters, the classical approach is to compute the actuator coordinates thanks to the complete inverse position relationship updated with the identified geometrical lengths:

$$
\begin{gathered}
\boldsymbol{q}=\boldsymbol{I P R}_{C}\left(\boldsymbol{x}, \widehat{\boldsymbol{k}_{C}^{b}}\right) \\
\widehat{\boldsymbol{k}^{b}}=\boldsymbol{k}_{C}^{b}+\widehat{\delta \boldsymbol{k}_{C}^{b}}
\end{gathered}
$$

where $\widehat{\delta \boldsymbol{k}_{C}^{b}}$ has been estimated during the identification process as explained previously, and $\boldsymbol{k}_{C}^{b}$ is the vector of nominal values of the base geometrical parameters. However the complete position relationship does not necessarily exist in a close form for parallelogram-based parallel robots. Therefore, instead of using a recursive algorithm (that might present some convergence problems or may be difficult to compute in real time), an approximation of the complete inverse position relationship $\widetilde{\boldsymbol{I P R}} C$ is proposed. In the following, the way to derive this approximated model will be explained. Starting from $\boldsymbol{x}$, the goal is to derive $\boldsymbol{q}$. Few steps are required:

(i) The simplified inverse position relationship using the nominal geometrical parameters, gives the positions of the actuators, as they were computed before calibration:

$$
\boldsymbol{q}=\boldsymbol{I P R _ { S }}\left(\boldsymbol{x}, \boldsymbol{k}_{S}\right) .
$$

(ii) Knowing $\boldsymbol{x}, \boldsymbol{q}$ and $\widehat{\delta \boldsymbol{k}_{C}^{b}}$, the identification model can be used in a straightaway manner in order to predict the positioning error $\delta \boldsymbol{X}$ of the TCP, which has been measured during the identification phase:

$$
\delta \boldsymbol{X}=\boldsymbol{S}_{C}^{b}\left(\boldsymbol{q}, \boldsymbol{k}_{C}\right) \widehat{\delta \boldsymbol{k}_{C}^{b}}
$$

(iii) Hence the part of it representing the translation errors of the TCP $\delta \boldsymbol{x}$ (corresponding to the operational coordinates $\boldsymbol{x}$ ), needs to be compensated in order for the robot to be perfectly accurate:

$$
\delta \boldsymbol{x}=\delta \boldsymbol{X}(1: 3) \text { (the three first elements) }
$$

(iv) The robot will have to move backward and make displacement $-\delta \boldsymbol{x}$, corresponding to a correction $-\delta \boldsymbol{q}$ of the actuators, in order to compensate the positioning error. As this displacement is very small, we can compute it making the linear approximation, that is to say using the classical jacobian matrix:

$$
\delta \boldsymbol{q}=J_{S}^{m}\left(\boldsymbol{q}, \boldsymbol{k}_{S}\right) \delta \boldsymbol{x}
$$

(v) Finally the value for $\boldsymbol{q}$ in order to compensate the positioning error of the TCP is obtained:

$$
\boldsymbol{q}_{\text {corrected }}=\boldsymbol{q}-\delta \boldsymbol{q} .
$$

Fig. 2 summarizes the whole process using a block diagram.

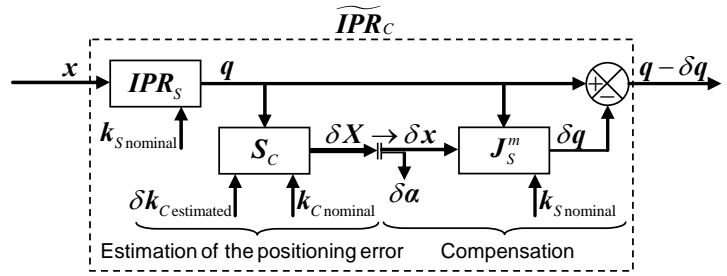

Fig. 2 - Computation scheme of the complete inverse position relationship's approximation 
To conclude, in the above parts, we have explained how to run a calibration process on a Delta robot, taking into account the complete geometry of the robot, including the exact geometry of the parallelograms. In the next section, we will enlighten the benefits of this method compared to classical ones, using a simple mechanism.

\section{ILLUSTRATION OF THE PROPOSED METHOD ON A SIMPLE MECHANISM}

This section illustrates the benefits of the proposed method using a simple mechanism. The taken mechanism was chosen because it is simple enough to derive all the required models in a close form, and complex enough to witness the properties of parallelogrambased parallel robots. It is a planar mechanism made with a parallelogram (see Fig. 3, left part). It has one degree of freedom: the revolute joint located at point $\mathrm{A}_{1}$ is actuated $\left(q+q_{0}\right)$, and the operational motion is the position of point $\mathrm{C}$ along $\vec{x}_{b}$. On the right part of Fig. 3, a mechanism corresponding to the virtual one used for computing the simplified models is shown. It consists in a bar $(l)$ actuated at point $\mathrm{O}_{\mathrm{b}}$ using a revolute joint $\left(q+q_{0}\right)$.
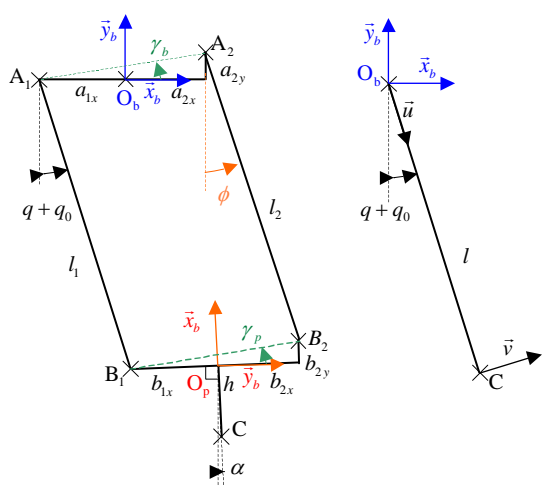

Fig. 3 - Scheme of the simple mechanism. Left: exact mechanism. Right: equivalent simplified mechanism used for the computation of the models

The strategy to illustrate the benefits of the proposed method is the following:

(i) To simulate a real mechanism, that is to say to introduce infinitesimal errors on all geometrical parameters,

(ii) To run an identification process on it,

(iii) To simulate some placements of the TCP after compensation,

(iv) To compute the positioning error once compensated using the different calibration strategies (see Fig. 4):

1.) No calibration, 2.) Classical calibration, 3.) Improved calibration.

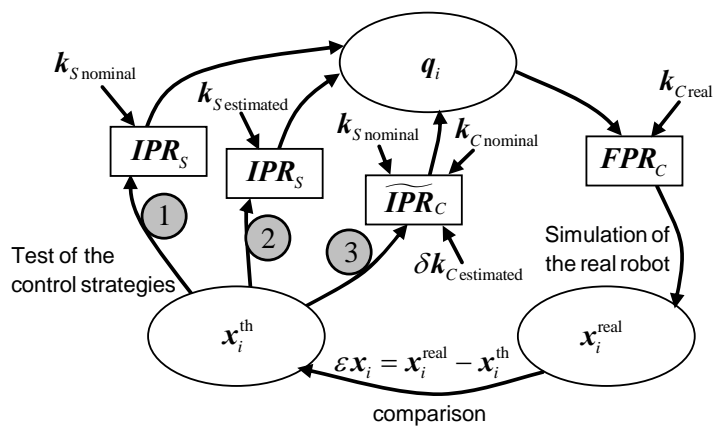

Fig. 4 - Scheme of the simulation process

\section{A. Modeling}

\section{1) Simplified models}

a) Position relations

The simplified forward position relationship is derived when projecting vector $\overrightarrow{\mathrm{O}_{\mathrm{b}} \mathrm{C}}$ along vector $\vec{x}_{b}$ :

$$
x=l \sin \left(q+q_{0}\right)
$$

This leads also to the inverse relationship:

$$
q=\arcsin (x / l)-q_{0}
$$

b) Velocity relations

Writing the derivative of relations (34) with respect to time leads to the forward simplified velocity relationship:

$$
\dot{x}=l \cos \left(q+q_{0}\right) \dot{q}
$$

Inverting (36) leads to the inverse relationship:

$$
\dot{q}=\frac{1}{l \cos \left(q+q_{0}\right)} \dot{x}
$$

\section{c) Geometrical calibration relations}

To compute the geometrical calibration model, the partial derivative of relation (34) with respect to the geometrical parameters is derived and the linear approximation (3) is done:

$$
\delta x=\left[\begin{array}{l:l}
l \cos \left(q+q_{0}\right) & \sin \left(q+q_{0}\right)
\end{array}\right]\left[\begin{array}{c}
\delta q_{0} \\
-\overline{\delta l}
\end{array}\right]
$$

\section{2) Complete Model}

In order to derive the complete model of the simple mechanism, the following frames, points, distances and angles have been defined (see Fig. 3):

$\square R_{b}\left(\mathrm{O}_{b}, \vec{x}_{b}, \vec{y}_{b}\right)$ : the frame located on the base, $\vec{x}_{b}$ is along $\left(\mathrm{O}_{b} A_{1}\right), \vec{y}_{b}$ is normal to $\vec{x}_{b}$,

$\square R_{p}\left(\mathrm{O}_{p}, \vec{x}_{p}, \vec{y}_{p}\right)$ : the frame attached to the platform, $\vec{x}_{p}$ is along $\left(O_{p} B_{1}\right)$ and $\vec{y}_{p}$ is normal to $\vec{x}_{p}$,

$\checkmark A_{1}$ and $A_{2}$ : the nominal centers of the revolute joints located on the base respectively defined such as $\overrightarrow{\mathrm{O}_{b} \mathrm{~A}_{1}}=-a_{1 x} \vec{x}_{b}$ and $\overrightarrow{\mathrm{O}_{b} \mathrm{~A}_{2}}=a_{2 x} \vec{x}_{b}+a_{2 y} \vec{y}_{b}$,

$\square B_{1}$ and $B_{2}$ : the nominal centers of the revolute joints located on the platform respectively defined such as $\overrightarrow{\mathrm{O}_{p} \mathrm{~B}_{1}}=-b_{1 x} \vec{x}_{p}$ and $\overrightarrow{\mathrm{O}_{p} \mathrm{~B}_{2}}=b_{2 x} \vec{x}_{p}+b_{2 y} \vec{y}_{p}$,

๑C : the TCP defined such as $\overrightarrow{\mathrm{O}_{p} \mathrm{C}}=-h \vec{y}_{p}$ $l_{1}$ and $l_{2}$ : the nominal lengths of rods $\mathrm{A}_{1} \mathrm{~B}_{1}$ and $\mathrm{A}_{2} \mathrm{~B}_{2}$, $\checkmark h$ : the nominal length between $\mathrm{O}_{p}$ and the TCP,

$\square q+q_{0}$ : the angle between the vector $\overrightarrow{\mathrm{A}_{1} \mathrm{~B}_{1}}$ and $\overrightarrow{y_{0}}$ that gives the actuated joint value.

The complete model that we used involves thus 10 geometrical parameters and the calibration purpose is then to identify the errors related to each parameter. However, it is to be noted that the error on the parameter $h$ will not be identified since it corresponds to the tool length which calibration is usually viewed as a metrology problem. Moreover, this model in not independent since it is obvious that some parameters are dependant of some others. This parameter dependency has been voluntary introduced in the model 
in order to verify the method explained in section (II-B-2) which allows to study the parameters observability.

The vector $\boldsymbol{k}_{C}$ including the 9 geometrical parameters is defined as follows:

$$
\boldsymbol{k}_{C}=\left[\begin{array}{l|lll:lll:ll}
q+q_{0} & a_{1 x} & a_{2 x} & a_{2 y} & b_{1 x} & b_{2 x} & b_{2 y} & l_{1} & l_{2}
\end{array}\right]^{T}
$$

and the vector $\delta \boldsymbol{k}_{C}$ of the geometrical errors that has to be identified is:

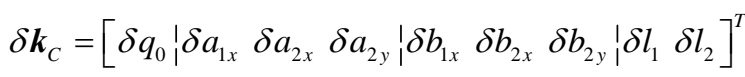

$$
\begin{aligned}
& \text { a) Position relations }
\end{aligned}
$$

Based on the parameterization previously defined, the Forward Position Relation for the Complete model (41) has to be derived.

$$
x=\boldsymbol{F P R}_{C}\left(q_{0}, \boldsymbol{k}_{C}\right)
$$

For this purpose, the following geometrical closure relations of the mechanism can be written (Figure 6):

$$
\begin{aligned}
& \overrightarrow{\mathrm{O}_{b} \mathrm{C}}=\overrightarrow{\mathrm{O}_{b} \mathrm{~A}_{1}}+\overrightarrow{\mathrm{A}_{1} \mathrm{~B}_{1}}+\overrightarrow{\mathrm{B}_{1} \mathrm{O}_{p}}+\overrightarrow{\mathrm{O}_{p} \mathrm{C}} \\
& \overrightarrow{\mathrm{O}_{b} \mathrm{C}}=\overrightarrow{\mathrm{O}_{b} \mathrm{~A}_{2}}+\overrightarrow{\mathrm{A}_{2} \mathrm{~B}_{2}}+\overrightarrow{\mathrm{B}_{2} \mathrm{O}_{p}}+\overrightarrow{\mathrm{O}_{p} \mathrm{C}}
\end{aligned}
$$

Subtracting (42) to (43) and projecting the resulting relation respectively onto $\vec{x}_{b}$ and $\vec{y}_{b}$ axes leads to the following equations:

$$
\begin{aligned}
\sin (\phi) & =E+F \cos (\alpha)+G \sin (\alpha) \\
\cos (\phi) & =E^{\prime}+G \cos (\alpha)-F \sin (\alpha)
\end{aligned}
$$

The angle $\alpha$ defines the platform orientation with respect to the base $\vec{x}_{p}$ axis and $\phi$, the angle between the vector $\overrightarrow{\mathrm{A}_{2} \mathrm{~B}_{2}}$ and $\overrightarrow{y_{b}}$ axis (Figure 6). The variables $E, E^{\prime}, F$ and $G$ can be calculated by using the following relations:

$$
\begin{gathered}
E=\frac{1}{l_{2}}\left(l_{1} \sin \left(q+q_{0}\right)-\left(a_{1 x}+a_{2 x}\right)\right) \\
E^{\prime}=\frac{1}{l_{2}}\left(l_{1} \cos \left(q+q_{0}\right)+a_{2 y}\right) \\
F=\frac{1}{l_{2}}\left(b_{1 x}+b_{2 x}\right), G=\frac{1}{l_{2}}\left(h-b_{2 y}\right)
\end{gathered}
$$

The following relation obtained by means of the law of cosines applied to triangles $A_{1} A_{2} B_{1}$ and $A_{2} B_{2} B_{1}$ is then set:

$$
\begin{aligned}
& A_{1} A_{2}^{2}+A_{1} B_{1}^{2}-2 A_{1} A_{2} A_{1} B_{1} \cos \left(\overline{\left(\overline{A_{1} A_{2}}, \overline{A_{1} B_{1}}\right.}\right) \\
& =A_{2} B_{2}^{2}+B_{2} B_{1}^{2}-2 A_{2} B_{2} B_{2} B_{1} \cos \left(\overline{\overline{A_{1} B_{2}}, \overline{B_{2} B_{1}}}\right)
\end{aligned}
$$

Merging the values of $\sin (\phi)$ and $\cos (\phi)$ defined by (44) and (45) within (49) allows rewriting that last equation under the following form:

$$
E^{\prime} \sin \left(\alpha+\gamma_{p}\right)-E \cos \left(\alpha+\gamma_{p}\right)=I
$$

The variable $I$ is obtained as follows:

$$
I=\frac{l_{b}^{2}-l_{p}^{2}+l_{1}^{2}-l_{2}^{2}+2 l_{b} l_{1} \sin \left(\gamma_{b}-q\right)}{2 l_{p} l_{2}}+F \cos \left(\gamma_{p}\right)+G \sin \left(\gamma_{p}\right)
$$

with (see Fig. 3):

$\square \gamma_{b}$ : the angle between $\overrightarrow{\mathrm{A}_{1} \mathrm{~A}_{2}}$ and $\overrightarrow{x_{b}}$,

$\checkmark l_{b}$ : the distance between $A_{1}$ and $A_{2}$,

$\square \gamma_{p}$ : the angle between $\overrightarrow{\mathrm{B}_{1} \mathrm{~B}_{2}}$ and $\overrightarrow{x_{b}}$,

$\checkmark l_{p}$ : the distance between $\mathrm{B}_{1}$ and $\mathrm{B}_{2}$.

From equation (50), the value of the platform orientation angle can be calculated as follows:

$$
\alpha=\arctan \left(\frac{E^{\prime} I-E \sqrt{E^{\prime 2}+E^{2}-I^{2}}}{E I+E^{\prime} \sqrt{E^{\prime 2}+E^{2}-I^{2}}}\right)-\gamma_{b}
$$

The value of $\alpha$ is then replaced in equation (42) whose projection onto $\overrightarrow{x_{b}}$ axis gives the value of $x$ that is the solution of the Forward Position Relationship (41).

\section{b) Velocity relations}

The complete velocity relations $\left(\dot{\boldsymbol{q}}=\boldsymbol{J}_{C}^{m} \dot{\boldsymbol{x}}\right.$ and $\left.\dot{\boldsymbol{x}}=\boldsymbol{J}_{C} \dot{\boldsymbol{q}}\right)$ are not required to run the calibration method. However, they could be derived straightforwardly, for example when computing the partial derivative of the inverse position relationship with respect to $\boldsymbol{x}$ :

$$
\begin{gathered}
\boldsymbol{J}_{C}^{m}=\frac{\partial \boldsymbol{I P \boldsymbol { R } _ { C } ( x , \boldsymbol { k } _ { C } )}}{\partial \boldsymbol{x}} \\
\boldsymbol{J}_{C}=\left(\boldsymbol{J}_{C}^{m}\right)^{-1}
\end{gathered}
$$

\section{c) Geometrical calibration relations}

To compute the sensibility matrix involved during calibration, the same method used for parallelogram-based parallel robots is used: instead of writing the partial derivative of the position relationships with respect to the geometrical parameters, the system made with equations ${\overrightarrow{\mathrm{A}_{i} \mathrm{~B}_{i}}}^{2}=l_{i}^{2}$ is taken. However, this system is not sufficient to compute the complete geometrical relationships, as the travelling plate has three dof when considered alone in the planar space, that need to be constrained by the mechanism. Thus a third relation to the calibration model is added. It is the linear relation linking the offset error of the actuator to the small displacement of point $\mathrm{B}_{1}$ when defined relatively to the TCP:

$$
d \overrightarrow{\mathrm{B}_{1}} \cdot \vec{v}_{1}=l_{1} d q_{0}
$$

$\vec{v}_{1}$ is the unitary vector perpendicular to vector $\overrightarrow{\mathrm{A}_{1} \mathrm{~B}_{1}}$ (see Fig. 3, left part). It is to note that, such an extra relation is useless when modeling a Delta robot, as there is as many equations as 6 , the number of degrees of freedom of the traveling plate when considered isolated into space.

Once written in the canonic basis $\mathcal{B}=\left(\vec{x}_{b}, \vec{y}_{b}\right)$, and making the linear approximation (3), the system made with these three equations becomes:

$$
\left\{\begin{array}{r}
\mathbf{A}_{1} \mathbf{B}_{1}{ }^{\mathrm{T}}\left(\delta \mathbf{B}_{1}-\delta \mathbf{A}_{1}\right)-l_{1} \delta l_{1}=0 \\
\mathbf{A}_{2} \mathbf{B}_{2}{ }^{\mathrm{T}}\left(\delta \mathbf{B}_{2}-\delta \mathbf{A}_{2}\right)-l_{2} \delta l_{2}=0 \\
\boldsymbol{v}_{1}{ }^{\mathrm{T}} \delta \mathbf{B}_{1}-l_{1} \delta q_{0}=0
\end{array}\right.
$$

Making the small displacements assumption $\delta \mathbf{B}_{i}$ can be written:

$$
\delta \mathbf{B}_{i}=\delta \boldsymbol{x}+\widehat{\mathbf{B}_{i} \mathbf{C}} \delta \alpha+\delta \boldsymbol{b}_{i}, i \in\{1,2\}
$$

where $\widehat{\mathbf{B}_{i} \mathbf{C}}$ represents the planar cross-product matrix, and $\delta \boldsymbol{b}_{i}$ corresponds to position errors of point $\mathrm{B}_{i j}$ relatively to the traveling plate. As points $A_{i}$ are connected directly to the ground, the following relations are derived:

$$
\delta \mathbf{A}_{i}=\delta \boldsymbol{a}_{i}, i \in\{1,2\}
$$

$\delta \boldsymbol{a}_{i}$ is the position error of point $\mathrm{A}_{i}$ relatively to the ground.

As usually done for calibration the taken values for the geometrical parameters during identification are the nominal values. Hence:

$$
l_{1}=l_{2}=l
$$


and vectors $\mathbf{A}_{1} \mathbf{B}_{1}, \mathbf{A}_{2} \mathbf{B}_{2}$ can be computed using the simplified

model:

$$
\mathbf{A}_{1} \mathbf{B}_{1}=\mathbf{A}_{2} \mathbf{B}_{2}=l \boldsymbol{u}
$$

with:

$$
\boldsymbol{u}=\left[\begin{array}{l}
u \\
v
\end{array}\right]=\left[\begin{array}{c}
\sin \left(q_{0}+q\right) \\
-\cos \left(q_{0}+q\right)
\end{array}\right]
$$

As $\boldsymbol{v}_{1}$ is the unitary vector orthogonal to $\mathbf{A}_{1} \mathbf{B}_{1}$, it can be defined using $u$ and $v$ :

$$
\boldsymbol{v}_{1}=\boldsymbol{v}=\left[\begin{array}{ll}
-v & u
\end{array}\right]^{\mathrm{T}}
$$

Hence, system (56) can be written in a synthetic manner:

$$
\boldsymbol{J}_{X} \delta \boldsymbol{X}=\boldsymbol{J}_{k C} \delta \boldsymbol{k}_{C}
$$

with:

$$
\boldsymbol{J}_{X}=\left[\begin{array}{ccc}
u & v & -u h-v b_{1 x} \\
u & v & -u h+v b_{2 x} \\
-v & u & v h-u b_{1 x}
\end{array}\right]
$$

and: $\quad \boldsymbol{J}_{k C}=\left[\begin{array}{c:ccc:ccc:cc}0 & -u & 0 & 0 & u & 0 & 0 & 1 & 0 \\ 0 & 0 & u & v & 0 & -u & -v & 0 & 1 \\ -l & 0 & 0 & 0 & -v & 0 & 0 & 0 & 0\end{array}\right]$

At the end, the sensibility matrix mapping the positioning error of the TCP $\delta \boldsymbol{X}$ to the errors on geometrical parameters $\delta \boldsymbol{k}_{C}$ is obtained:

$$
\delta \boldsymbol{X}=\boldsymbol{S}_{C} \delta \boldsymbol{k}_{C}
$$

with:

$$
\boldsymbol{S}_{C}=\boldsymbol{J}_{X}{ }^{-1} \boldsymbol{J}_{k C},
$$

$$
\delta \boldsymbol{k}_{C}=\left[\begin{array}{l|lll|lll|ll}
\delta q_{0} & \delta a_{1 x} & \delta a_{2 x} & \delta a_{2 y} & \delta b_{1 x} & \delta b_{2 x} & \delta b_{2 y} & \delta l_{1} & \delta l_{2}
\end{array}\right]^{\mathrm{T}}
$$

\section{B. Comparison results}

Simulations have been performed in order to verify the efficiency of the proposed method. For this purpose, a set of geometrical error and parameter values has been chosen to simulate the classical and proposed calibration method of the introduced simple mechanism (see TABLE 2).

According to the calibration relations developed in previous sections, for both simplified and complete models, the identification of the geometrical errors have been performed by using 19 measurement configurations corresponding to $q$ values varying from $-45^{\circ}$ to $45^{\circ}$ with $5^{\circ}$ steps. For each configuration, we made the assumption that complete measurements were available (position and orientation). Moreover, we defined for simulations Gaussian measuring noises with standard deviations of $0.003 \mathrm{~mm}$ and $0.003^{\circ}$ respectively.

TABLE 2

GEOMETRICAL VALUES FOR THE COMPLETE AND SIMPLIFIED MODEL

\begin{tabular}{|l|l|l|}
\hline Parameter nominal values & Error values & Unit \\
\hline$q \in\left[-45^{\circ}, 45^{\circ}\right] / q_{0}=0^{\circ}$ & $\delta q_{0}=0.01^{\circ}$ & deg \\
\hline$a_{1 x}=125$ & $\delta a_{1 x}=1$ & $\mathrm{~mm}$ \\
\hline$a_{2 x}=125, a_{2 y}=0$ & $\delta a_{2 x}=0, \delta a_{2 y}=3$ & $\mathrm{~mm}$ \\
\hline$b_{1 x}=125$ & $\delta b_{1 x}=0$ & $\mathrm{~mm}$ \\
\hline$b_{2 x}=125, b_{2 y}=0$ & $\delta b_{2 x}=-2, \delta b_{2 y}=0$ & $\mathrm{~mm}$ \\
\hline$l_{1}=l_{2}=l=950$ & $\delta l_{1}=1, \delta l_{2}=-1$ & $\mathrm{~mm}$ \\
\hline$h=100$ & --- & $\mathrm{mm}$ \\
\hline
\end{tabular}

For the complete model identification, the observability study showed that only 6 over the 9 geometrical errors could be identified. As a result, the parameters $\delta a_{2 x}, \delta a_{2 y}$ and $\delta b_{1 x}$ have been identified as linear combinations of others according to the following obvious relationships:

$$
\begin{aligned}
\delta a_{2 x I} & =\delta a_{2 x}-\delta b_{2 x} \\
\delta a_{2 y I} & =\delta a_{2 y}-\delta b_{2 y} \\
\delta b_{1 x I} & =\delta b_{1 x}-\delta a_{1 x}
\end{aligned}
$$

Hence, the base of identifiable geometrical errors that have been used to correct the complete model is:

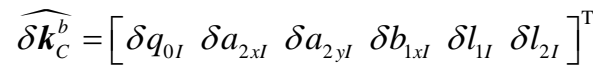

In the same manner, for the simplified model, the vector of identified geometrical errors is noted:

$$
\widehat{\delta \boldsymbol{k}_{S}}=\left[\begin{array}{ll}
\delta q_{0 I} & \delta l_{I}
\end{array}\right]^{\mathrm{T}}
$$

The proposed compensation method has been run by using $\widehat{\delta \boldsymbol{k}_{C}^{b}}$ and $\widehat{\delta \boldsymbol{k}_{S}}$ (Fig. 3). Results given on Fig. 5, Fig. 6 and Fig. 7 show the TCP positioning errors for the simple mechanism with 1.) no calibration and after 2.) classical and 3.) improved calibration methods (see Fig. 4). These errors correspond to the values of $q$ varying from $-45^{\circ}$ to $45^{\circ}$ with $1^{\circ}$ steps. They have been evaluated with tool lengths $h=0$, and $\mathrm{h}=200 \mathrm{~mm}$. As it can be noted, the proposed calibration method allows to compensate efficiently the TCP positioning errors due to geometrical errors of the models (green curve). This compensation is much more reliable than the one achieved with the classical approach (blue curve) since it does take into account the whole geometry of the parallelogram. Moreover, as one can see, this difference is accentuated when the value of $h$ increases. This amplifies the effects of the geometrical errors on the platform orientation and thus on the resulting TCP positioning errors. Another remark concerns the values of the TCP positioning errors remaining after running the improved calibration process which are directly linked to the identification errors of $\delta \boldsymbol{k}_{C}^{b}$ during least squares estimation. As it is well-known, these estimation errors could be reduced by reducing the measuring noise or by increasing the number of measurement configurations (see Fig. 7).

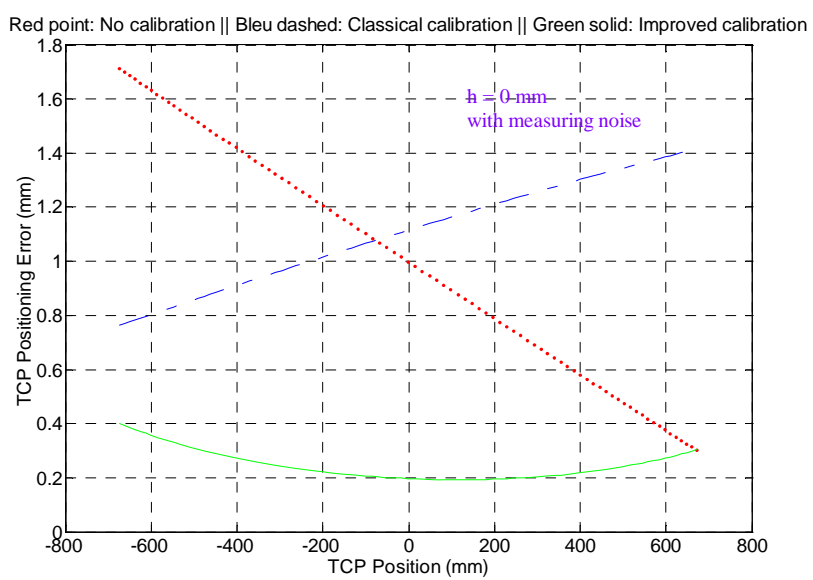

Fig. 5 - TCP positioning before and after classical and improved calibration 


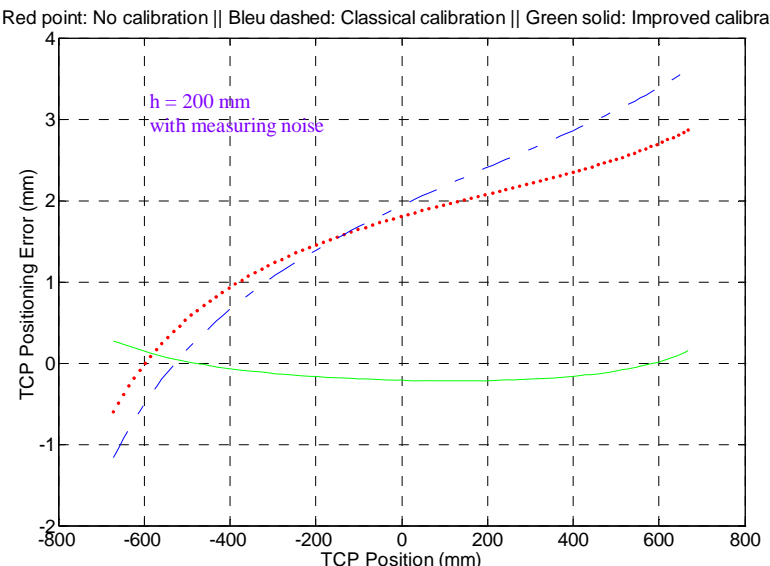

Fig. 6 - TCP positioning before and after classical and improved calibration

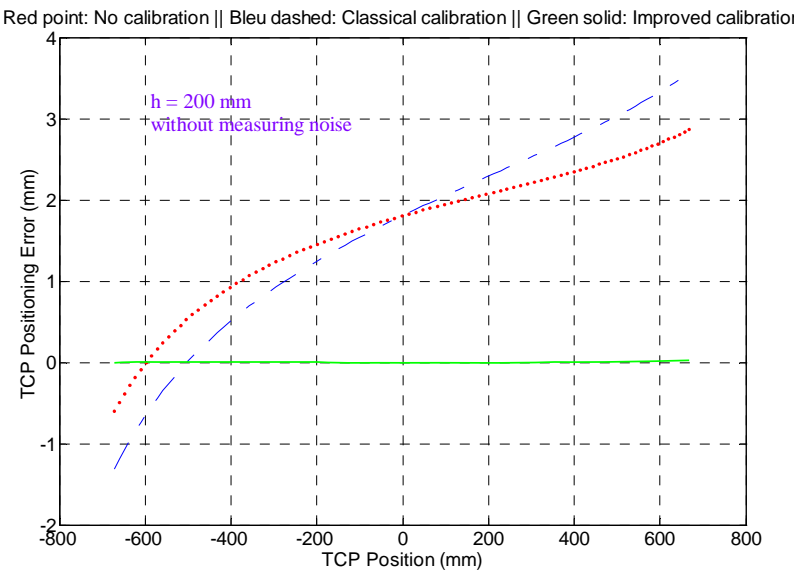

Fig. 7 - TCP positioning before and after classical and improved calibration.

\section{FURTHER WORK AND CONCLUSION}

The work presented in this paper focuses on the improvement of the static accuracy of the PKM family for which the structure is based upon some parallelogram mechanisms. For this purpose an improved calibration method has been developed in order to compensate the TCP positioning accuracy errors of those machines. The originality of that method in comparison with those that have been already proposed consists in identifying the whole geometry of the PKM structure including thus the entire parallelogram geometry. That identification combined with a proposed compensation strategy allows to correct efficiently the effects of the infinitesimal rotations of the traveling plate induced by the parallelogram geometrical errors. The compensation strategy is achieved with the linear approximation model of the machine positioning relationships that is derived and calculated by including the identified geometrical errors obtained during calibration.

The main principle of the proposed calibration method has been explained by applying it on the well-know Delta robot for which the calibration relations and the compensation scheme have been described. This has been done in order to show how it can be also applied to other parallelogram-based PKM.
Then its efficiently has been shown by involving it to improve the static accuracy of a simple 1-dof planar mechanism, simple enough to derive all calibration models in a close form and complex enough to witness the properties of parallelogram-based parallel robots. Future works concern the first experimental tests that will be achieved on the Delta robot of our laboratory. Then the objective will be to include within the calibration models of the proposed calibration method, the static elastic deformations of the PKM structure under the gravity effects. Finally, our purpose will be also to apply the proposed method to other complex parallel manipulators where error in parameters is an important issue and for which this method becomes useful especially when the complete position relationships cannot be derived in a close form.

\section{REFERENCES}

[1] Grossmann K., Wunderlich B., Szatmari S., "Progress in accuracy and calibration of parallel kinematics,” Proc. Parallel Kinematics Seminar 2004, pp 49-68, Chemmnitz, Germany, April 2004.

[2] Rauf A., Kim S.-G, Ryu J., "A new measurement device for complete parameter identification of parallel manipulators with partial pose measurements," Proc. Parallel Kinematics Seminar 2004, pp 89-105, Chemmnitz, Germany, April 2004.

[3] Weck M., Staimer D., “Accuracy Issues of Parallel Kinematic Machines: Design calibration and compensation," In Production Engineering VIII/2 2001, pp 99-102.

[4] Weck M., Staimer D., "Parallel Kinematic Machine Tools - Current state and future potentials," In Annals of the CIRP, Vol 51/2/2002, pp 1-13.

[5] Pristchow G., "Parallel kinamatic machine tools - Limitations and new solutions,” In Annals of the CIRP, Vol 49/1/2000, pp 275-280.

[6] Gosselin C. M., Zhang D., "Stiffness analysis of parallel mechanisms using a lumped model," International Journal of Robotics and Automation, vol 17, no. 1, pp17-27, 2002

[7] Majou F., Gosselin C., Wenger P., Chablat D., "Parametric stiffness analysis of the Orthoglide", Proc. The 35th International Symposium on Robotics, Paris, France, March 2004.

[8] Ecorchard G, Maurine P. "Self-Calibration of Delta Parallel Robots with Elastic Deformation Compensation,” Proc. IROS 2005, Canada, 2005.

[9] Clavel, R., "Device for the Movement and Positioning of an Element in Space,” US Patent No. 4976 582, December 11, 1990.

[10]F. Pierrot, O. Company and F. Marquet, "H4: a high speed 4dof parallel robot. Synthesis, Modelling and Control Issues," in IEEE Transactions on Robotics and Automation, Vol. 19, No 3, pp.411-420, June 2003

[11]Bruyninckx H , "The 321-hexa: a fully parallel manipulator with closedform position and velocity kinematics," In IEEE Int. Conf. On Robotics and Automation, pages 2657-2662, Albuquerque, 21-28 Avril 1997

[12]Zobel P.B. and Clavel R., "On the static calibration of the Delta parallel robot,” IASTED Robotics and Manufacturing, Oxford, 1993, pp 88-91.

[13] Vischer P., Clavel R. "Kinematic calibration of the parallel Delta robot", Robotica, 16(2),pp. 207-218, March- April 1998.

[14]Baradat C., Deblaise D., Bazin E., Maurine P. "Artefact Based Calibration and Verification of PKM," Proc. The 35th International Symposium on Robotics, Paris, France, March 2004.

[15]Deblaise D. Maurine P. "Effective Geometrical Calibration of a Delta Parallel Robot Used in Neurosurgery,” Proc. IROS 2005, Canada, 2005.

[16]B. Mooring, Z. Roth, M. Driels , "Fundamentals of manipulator calibration,” John Wiley \& Sons Inc., New York, 1991.

[17]Wang J., "Workspace Evaluation and Kinematic Calibration of Stewart Platform,” Ph.D. dissertation, Florida Atlantic University, FL 1992.

[18]Besnard S., Khalil W., "Identifiable parameters for parallel robots kinematic calibration," Proc. IEEE Int. Conference Robotics and Automation, pp. 2859-2866, Seoul, Korea, May 2001.

[19]Besnard S., "Etalonnage géométrique des robots série et parallèles," $P h D$ Thesis, Ecole Centrale de Nantes, Nantes, France 2000. 